\title{
Programa Patrulha da Saúde: indicadores de saúde em policiais rodoviários federais
}

\author{
Health's Patrol Program: health indicators from federal highway policemen
}

\author{
Eduardo Frio Marins ${ }^{1,2} \bowtie$, Fabrício Boscolo Del Vecchio ${ }^{1}$
}

1 Escola Superior de Educação Física da Universidade Federal de Pelotas (UFPel). Pelotas, RS.

2 Departamento de Polícia Rodoviária Federal (DPRF). 9a Superintendência Regional de Polícia Rodoviária Federal no Rio Grande do Sul. Pelotas, RS.

\section{RESUMO}

OBJETIVOS: Avaliar o perfil sociodemográfico, de saúde, nutricional, físico e funcional de policiais rodoviários federais participantes do Programa Patrulha da Saúde e avaliar a associação dessas características com idade e tempo de serviço.

MÉTODOS: Estudo transversal com policiais rodoviários federais de Pelotas, Rio Grande do Sul, em setembro de 2015. Foram incluídos participantes do Programa Patrulha da Saúde que não estavam de férias ou licença. Aplicou-se questionário com informações sociodemográficas, nível de atividade física e sintomas osteomusculares. Foram aferidos dados antropométricos e nutricionais, pressão arterial e capacidades físicas e funcionais. As variáveis foram categorizadas por idade e tempo de serviço. Utilizaram-se os testes t independente e Qui-quadrado ou exato de Fisher para avaliar as associações. O nível de significância adotado foi $\mathrm{p} \leq 0,05$.

RESULTADOS: Dos 64 policiais da delegacia de Pelotas, 41 (64,1\%), todos homens, foram incluídos no estudo. Houve percentuais superiores de casados, não fumantes, com pressão arterial normal e fisicamente ativos no lazer. Registraram-se as seguintes médias: índice de massa corporal 28,5 $\pm 3,4 \mathrm{~kg} / \mathrm{m}^{2}$, circunferência da cintura $99,2 \pm 8,5 \mathrm{~cm}$, gordura corporal $25,1 \pm 4,9 \%$, flexibilidade $21,2 \pm 9,4 \mathrm{~cm}$, força isométrica de preensão manual direita e esquerda respectivamente $47,4 \pm 6,2 \mathrm{kgf}$ e $44,6 \pm 6,3 \mathrm{kgf}$ e força isométrica de membros inferiores $127,1 \pm 20,8 \mathrm{kgf}$. O escore médio da avaliação funcional foi de $13,8 \pm 2,6$ pontos $(57 \%$ com $\leq 14$ pontos). Os sintomas osteomusculares que mais se associaram a afastamento do trabalho foram localizados no joelho $(22 \%)$ e lombar $(14,6 \%)$. Policiais mais velhos exibiram piores valores para índice de massa corporal, circunferência de cintura e porcentagem de gordura corporal, e os mais antigos na corporação demonstraram maior circunferência de cintura.

CONCLUSÕES: Os policiais rodoviários federais participantes do Programa Patrulha da Saúde em Pelotas, mesmo declarando estarem fisicamente ativos, apresentaram frequentemente condições morfológicas e físicas inadequadas, assim como níveis de força e flexibilidade inferiores aos recomendados para essa população. Nesta amostra, a idade mais avançada e maior tempo de serviço na Polícia Rodoviária Federal estiveram associados a parâmetros morfológicos de alto risco para doenças cardiovasculares. Observou-se, também, que os PRF possuíam alto risco de lesões musculoesqueléticas e afastavam-se do trabalho principalmente por queixas osteomusculares nas regiões lombar e dos joelhos.

DESCRITORES: promoção da saúde; policiais; aptidão física; atividade motora.

\section{ABSTRACT}

AIMS: To evaluate the socio-demographic, health, nutritional, physical and functional profile of federal highway policemen participants of the Health Patrol Program and evaluate the association of these characteristics with age and time of service.

METHODS: Cross-sectional study with federal highway police officers from the city of Pelotas, Rio Grande do Sul, Brazil, in September 2015. Health Patrol Program's participants who were not on vacation or license were included. Questionnaires with sociodemographic information, level of physical activity and musculoskeletal symptoms were applied. Anthropometric and nutritional data, blood pressure and physical and functional capacities were measured. The variables were categorized by age and time of service. The independent t-test and chi-square test or Fisher's exact test were used to evaluate the associations. The significance level adopted was $\mathrm{p} \leq 0.05$.

RESULTS: Of the 64 police officers from the Pelotas police station, 41 (64.1\%) men met the inclusion criteria. There were higher percentages of married, non-smokers, with normal blood pressure and physically active in leisure $(89.5 \%)$. The following means were recorded: body mass index $28.5 \pm 3.4 \mathrm{~kg} / \mathrm{m}^{2}$, waist circumference $99.2 \pm 8.5 \mathrm{~cm}$, body fat $25.1 \pm 4.9 \%$, flexibility $21.2 \pm 9.4 \mathrm{~cm}$, isometric handgrip strength of right and left hand respectively $47.4 \pm 6.2 \mathrm{kgf}$ and $44.6 \pm 6.3 \mathrm{kgf}$ and isometric strength of lower limbs $127.1 \pm 20.8 \mathrm{kgf}$. The average score of the functional evaluation was $13.8 \pm 2.6$ points $(57 \%$ with $\leq 14$ points). The musculoskeletal symptoms that were most associated with absence from work were located in knee (22\%) and low back (14,6\%). Older policemen exhibited higher values for body mass index, waist circumference and body fat percentage, and the oldest in the corporation showed greater waist circumference.

CONCLUSIONS: Even when they stated being physically active, federal highway police officers participating in the Health Patrol Program in Pelotas had often inadequate morphological and physical conditions, as well levels of strength and flexibility lower than those recommended for this population. In this sample, the more advanced age and longer service time in the Federal Highway Police were associated with morphological parameters of high risk for cardiovascular diseases. It was also observed that federal highway policemen had a high risk of musculoskeletal injuries and were absence from work mainly due to musculoskeletal complaints in the low back and knee regions.

KEY WORDS: health promotion; law enforcement officers; physical fitness; motor activity.

Recebido: novembro, 2016

Aceito: maio, 2017

Publicado: junho, 2017

Correspondência: eduardo.marins@prf.gov.br/eduardo_ifm@ufpel.edu.br

Universidade Federal de Pelotas - Escola Superior de Educação Física (ESEF)

Rua Luiz de Camões, 625 - CEP 96055-630, Pelotas, RS, Brasil
Este artigo está licenciado sob forma de uma licença Creative Commons Atribuição 4.0 Internacional, que permite uso irrestrito, distribuição e reprodução em qualquer meio, desde que a publicação original seja corretamente citada. http://creativecommons.org/licenses/by/4.0/deed.pt_BR 
Abreviaturas: AF, atividade física; $\mathrm{CC}$, circunferência da cintura; IMC, índice de massa corporal; FMS, Functional Movement Screen; MMII, membros inferiores; NAF, nível de atividade física; $\% \mathrm{GC}$, porcentagem de gordura corporal; PM, policiais militares; PPS, Programa Patrulha da Saúde; PRF, policiais rodoviários federais.

\section{INTRODUÇÃO}

A atividade policial é estressante e perigosa, uma vez que seus profissionais lidam, cotidianamente, com situações de violência, acidentes e morte, e ao mesmo tempo são submetidos a turnos de trabalho extenuantes, de longa duração [1]. Além disso, o trabalho policial, ocasionalmente, envolve o desempenho de tarefas físicas extremamente exigentes, como perseguir suspeitos em fuga, saltar obstáculos, controlar a resistência à prisão e deslocar indivíduos acidentados $[2,3]$. Destaca-se, ainda, que esses profissionais executam suas atividades transportando cargas com equipamentos de proteção que podem ultrapassar $20 \mathrm{~kg}$ [3]. Nesse contexto geral, o labor policial vem sendo associado à alta prevalência de doenças cardiovasculares, inclusive a síndrome metabólica, e a lesões musculoesqueléticas, gerando, assim, incremento nos casos de absenteísmo, morbidade e incapacidade [4-7].

No Brasil, a quantidade de benefícios concedidos pelo Instituto Nacional do Seguro Social em 2014, como auxílio doença e aposentadoria por invalidez, em virtude de distúrbios musculoesqueléticos (artrose, artrite, rupturas ligamentares), alcançou $18,5 \%$ dos casos, o que impacta significativamente na economia do País, com gastos próximos a R $\$ 534,5$ milhões [8]. Quando esses benefícios se referem a policiais, o controle social e a segurança da população ficam prejudicados, em decorrência do menor efetivo nas ruas e rodovias.

No cenário internacional são identificados programas de promoção à saúde de agentes de segurança pública $[9,10]$. Em contrapartida, pouco se sabe sobre programas institucionais que avaliem o perfil de saúde de policiais brasileiros, procedimento que poderia auxiliar no desenvolvimento de intervenções estimuladoras do comportamento ativo em policiais. É de grande valia a capacidade de identificar prejuízos à saúde, riscos de futuras lesões musculoesqueléticas, limitações e assimetrias individuais, assim como de conhecer o perfil profissional desses agentes da segurança pública $[1-3,5,6,11]$.
Na Polícia Rodoviária Federal, o Programa Patrulha da Saúde (PPS) é uma das ações realizadas com a finalidade de avaliar capacidades físicas e promover conhecimento sobre medidas de prevenção a doenças, assim como uma estratégia de aumentar a conscientização para prática de hábitos saudáveis no ambiente policial e fora dele. O PPS consiste em um conjunto de avaliações que caracterizam o perfil sociodemográfico, de saúde, funcional e físico dos policiais rodoviários federais (PRF). O PPS visa também combater os fatores de risco associados ao trabalho policial, como práticas alimentares inadequadas e inatividade física, prevenindo lesões musculoesqueléticas, doenças cardiovasculares, distúrbios do sono e incapacidade prematura.

Apesar do PPS estar sendo implementado nas diferentes regiões do Brasil, ainda se conhece pouco sobre as características dos PRF avaliados pelo mesmo, existindo uma lacuna na literatura acerca do assunto. Logo, os objetivos deste estudo foram descrever, analisar e classificar o perfil de saúde de PRF participantes do PPS e avaliar características antropométricas, de aptidão, nutricionais e funcionais desses profissionais em relação à idade e tempo de serviço.

\section{MÉTODOS}

Foi realizado um estudo transversal que incluiu PRF atuantes na 7ạ Delegacia da Polícia Rodoviária Federal, em Pelotas, Rio Grande do Sul. Foram adotados os seguintes critérios de inclusão: não estar em período de férias ou licença; ser PRF em efetivo exercício (operacional ou administrativo); e ter participado do PPS aplicado na 7a delegacia em 2015. Este estudo foi realizado dentro dos padrões éticos exigidos pelo Conselho Nacional de Saúde, em sua resolução no 466/12, e foi submetido e aprovado pelo Comitê de Ética e Pesquisa da Faculdade de Educação Física da Universidade Federal de Pelotas pelo parecer de número 005/2012.

Para a coleta de dados, doze avaliadores passaram por um dia prévio de capacitação, sendo dividido em parte teórica com professor doutor em educação física e atividade prática com acadêmicos do curso de pósgraduação em educação física. Posteriormente, os avaliadores foram alocados em cinco estações, sendo dois ou três deles em cada estação com um profissional especialista supervisor.

Os dados foram coletados em dois dias subsequentes, em horários pré-determinados, em setembro de 2015 , em um salão localizado nas proximidades da delegacia. Os dados foram obtidos por meio de 
questionários, avaliação antropométrica, avaliação nutricional, pressão arterial, avaliação motora e avaliação funcional.

\section{Questionários}

Os questionários foram divididos em dois blocos, sendo o primeiro previamente criado pela Polícia Rodoviária Federal para o PPS. Esse instrumento incluiu informações sobre sexo, idade, situação conjugal, tempo de serviço na Polícia Rodoviária Federal, presença de tabagismo e alcoolismo, auto percepção de saúde e auto relato sobre presença ou ausência de diabetes, hipertensão, doença cardíaca, pulmonar ou renal. No segundo bloco foram aplicados dois questionários, validados previamente, autopreenchidos pelos participantes do estudo.

a) International Physical Activity Questionnaire (IPAQ), versão curta, traduzido e validado no Brasil [12]. Este instrumento, composto por oito questões abertas, permite estimar o nível de atividade física (NAF) geral em diferentes facetas da atividade física (AF) domésticas, de deslocamento, ocupacionais e de lazer - e de inatividade física (tempo em que o indivíduo permanece sentado em um dia típico de semana e no fim de semana), a partir do tempo semanal gasto na realização de $\mathrm{AF}$ de intensidade moderada a vigorosa. Foram consideradas as atividades realizadas na semana anterior ao preenchimento do questionário. $\mathrm{O}$ resultado se deu pela seguinte fórmula: $\mathrm{NAF}=\mathrm{dc} * \mathrm{mc}+\mathrm{dafm} * \mathrm{mafm}+((\mathrm{dafv} * \mathrm{mafv}) * 2)$, onde $\mathrm{dc}=$ dias de caminhada/semana; $\mathrm{mc}=$ minutos de caminhada/semana; dafm=dias de AF moderada/ semana; mafm=minutos de AF/semana; dafv=dias de $\mathrm{AF}$ vigorosa/semana; $\mathrm{mafv}=$ minutos de $\mathrm{AF}$ vigorosa/semana. Para interpretação, consideraram-se duas categorias: suficientemente ativo $(\geq 150$ minutos/ semana); e insuficientemente ativo $(<150$ minutos/ semana).

b) Questionário Nórdico de Sintomas Osteomusculares (QNSO), traduzido e validado no Brasil [13]. Consiste em escolhas múltiplas ou dicotômicas quanto à ocorrência de sintomas em 10 regiões anatômicas do corpo: pescoço/cervical; ombros; cotovelos; antebraços; punhos/mãos/dedos; região dorsal; região lombar; quadris/coxas; joelhos; e tornozelos/pés. O respondente deve relatar a ocorrência dos sintomas considerando os 12 meses e os sete dias precedentes à entrevista, bem como a ocorrência de afastamento das atividades rotineiras no último ano. Seu objetivo é mensurar os relatos de sintomas osteomusculares.

\section{Avaliações antropométrica e nutricional}

A massa corporal foi aferida com balança digital (Soehnle Professional, Brasil), com precisão de $0,01 \mathrm{~kg}$, e a estatura com estadiômetro (Standard, Sanny ${ }^{\mathrm{TM}}$, Brasil). O índice de massa corporal (IMC) foi calculado dividindo a massa (em $\mathrm{kg}$ ) pelo quadrado da estatura (em m), sendo classificado segundo a Organização Mundial da Saúde [14]. A circunferência da cintura (CC) foi medida em um plano horizontal, no centro entre a margem inferior das costelas e a borda superior da crista ilíaca, utilizando uma trena antropométrica metálica inelástica (Sanny ${ }^{\circledR}$, Brasil) com precisão de $0,1 \mathrm{~cm}$. A gordura corporal (GC) foi avaliada por bioimpedância bipolar vertical (Techline ${ }^{\circledR}$, Brasil) seguindo as seguintes recomendações prévias à avaliação [15]: (i) jejum de pelo menos quatro horas; (ii) abster-se de exercícios intensos nas últimas 24 horas; (iii) urinar 30 minutos antes; (iv) não consumir álcool nas últimas 48 horas; (v) não fazer uso de diuréticos sete dias antes. A mensuração foi feita pelo contato das mãos com os dois eletrodos acoplados diretamente ao aparelho, com o resultado mostrado diretamente no mesmo. O valor obtido foi classificado de acordo com os valores de referência [16].

\section{Pressão arterial}

A pressão arterial foi aferida por meio de um esfigmomanômetro aneróide (Vasquez-Lubry ${ }^{\mathrm{TM}}$, Alemanha) e um estetoscópio (Littman ${ }^{\circledR}$, EUA), com o indivíduo sentado, braço na posição supina e apoiado em uma mesa na altura do coração.

\section{Avaliação motora}

a) Flexibilidade dos isquiotibiais (banco de Wells) - avaliada pelo teste de Sentar e Alcançar. Seguindo padronização canadense para testes de avaliação da aptidão física [17], o teste é realizado em uma caixa com escala de $26 \mathrm{~cm}$ em seu prolongamento, sendo que o ponto zero se encontra na extremidade mais próxima do avaliado e o $26 \mathrm{~cm}$ coincide com o ponto de apoio dos pés. Iniciando com o indivíduo sentado no chão, pés descalços em contato com a caixa e os joelhos estendidos, realiza-se, com os ombros flexionados, os cotovelos estendidos e as mãos sobrepostas, uma flexão de tronco à frente devendo tocar o ponto máximo mais longínquo possível na escala, mantendo a posição por dois segundos. Foram realizadas três tentativas, sendo considerado o maior valor. Os indivíduos foram classificados segundo o Canadian Standardized Test of Fitness [17]. 
b) Força isométrica de preensão manual (dinamômetro manual) - utilizou-se um dinamômetro $\left(\right.$ Jammar $^{\circledR}$, EUA) de forma bilateral, conforme as recomendações da Sociedade Americana de Terapeutas de Mão. Na posição sentada, ombro aduzido, cotovelo flexionado a $90^{\circ}$ e antebraço em posição neutra, o indivíduo apertava o dinamômetro o mais forte que pudesse, realizando três tentativas em cada mão, intercalando os membros. O maior valor registrado em cada mão foi considerado. A classificação baseou-se nas diretrizes do American College of Sports Medicine [18] para teste de esforço.

c) Força isométrica de MMII (dinamômetro tóraco-lombar) - adotou-se dinamômetro $\left(\mathrm{CROWN}{ }^{\circledR}\right.$, Brasil) para aferição da força lombar e de MMII. Os policias posicionavam-se em pé na plataforma com os joelhos semiflexionados, tronco flexionado à frente formando um ângulo de aproximadamente $120^{\circ}$, cabeça no prolongamento do tronco com o olhar fixado à frente e os braços estendidos. A barra de apoio foi posicionada próxima à altura dos joelhos. O participante aplicou a maior força possível no movimento de extensão da coluna conjuntamente com os MMII. Foram classificados conforme as diretrizes do American College of Sports Medicine [18].

\section{Avaliação funcional}

Realizou-se o Functional Movement Screen (FMS), que é uma ferramenta de avaliação biomecânica de padrões de movimento básicos, mobilidade e estabilidade de regiões específicas, pela qual limitações e/ou assimetrias musculares são identificadas. Ela consiste em sete testes, realizados na seguinte sequência: overhead squat ou agachamento profundo; hurdle step ou passo sobre a barreira; inline lunge ou avanço em linha reta; shoulder mobility ou mobilidade de ombro; active straight-leg raise ou elevação da perna estendida; trunk stability push-up ou estabilidade de tronco; e rotary stability ou estabilidade de rotação. Em cada um deles é aplicada pontuação de 0 a 3. Essas pontuações são atribuídas com base nos seguintes critérios pré-estabelecidos: 3: movimento executado como previsto; 2: movimento executado com modificação ou imperfeição; 1: movimento não executado. Além disso é atribuída pontuação 0 a qualquer teste que gere dor, independentemente do desempenho do avaliado. A pontuação total do FMS pode variar de 0 a 21 [19]. A avaliação foi feita por dois avaliadores treinados e certificados para o respectivo teste.

\section{Análise estatística}

A análise de dados foi conduzida pelos testes de normalidade (Shapiro-Wilk) e homogeneidade (Bartlet). Após, foi efetivada a análise univariada dos dados a fim de descrever as variáveis categóricas (proporção e intervalo de confiança) e contínuas (médias e desvios padrão). Em relação à idade os participantes foram divididos em dois grupos: entre 30 e 41 anos e de 42 a 52 anos. Em relação ao tempo de serviço na Polícia Rodoviária Federal, também foi feita divisão em dois grupos: de 2 a 10 anos e de 11 a 21 anos. Assim, testes bivariados foram conduzidos com o teste $t$ independente para identificar diferenças nas médias entre os grupos. Os testes qui quadrado ou exato de Fischer foram utilizados para verificar a possível diferença na distribuição das variáveis categóricas nos dois grupos. Estabeleceu-se nível de significância estatística de 5\% e intervalo de confiança de $95 \%$. Os dados foram analisados utilizando o pacote estatístico Stata, versão 14.1.

\section{RESULTADOS}

Do total de 64 policiais lotados nessa delegacia, $43(67,2 \%)$ atendiam aos critérios de inclusão. Dentre estes, 41 (64,1\% entre os 64) eram homens e duas eram mulheres. Optou-se por incluir somente os homens devido ao pequeno número que constituiria o grupo de sexo feminino. Dessa forma, a análise incluiu apenas policiais do sexo masculino. A média de idade foi de $40,5 \pm 6,3$ anos e a média de tempo de serviço foi de $12,0 \pm 7,0$ anos.

Quando divididos nas categorias de idade e tempo de serviço, os policiais apresentaram as seguintes características: idade de 30 a 41 anos $(n=22$; média de $35,5 \pm 3,1$ anos) e de 42 a 52 anos ( $n=19$; média de $46,4 \pm 3,1$ anos); tempo de serviço de 2 a 10 anos ( $\mathrm{n}=22$; média de $6,4 \pm 3,3$ anos) e de 11 a 21 anos ( $\mathrm{n}=19$; média de 18,5 $\pm 3,5$ anos). Observa-se que embora os números de indivíduos nas categorias de idade e de tempo de serviço sejam iguais, os indivíduos não são os mesmos em cada categoria de cada variável.

A partir do teste de Shapiro-Wilk, todas as variáveis exibiam distribuição normal. Acerca da homogeneidade das variâncias, apenas IMC e \%GC portavam variâncias heterogêneas e, quando apropriado, foram consideradas nas interpretações.

As médias de massa corporal e estatura foram, respectivamente, de $88,7 \pm 12,0 \mathrm{~kg}$ e $1,76 \pm 0,06 \mathrm{~m}$. A estatura média dos mais jovens ( 30 a 41 anos; 
$1,78 \pm 0,06 \mathrm{~m})$ foi superior à dos mais velhos (42 a 52 anos; $1,74 \pm 0,05 \mathrm{~m})(\mathrm{p}=0,02)$.

A maior parte dos participantes eram casados ou viviam com companheira (75\%), 92,7\% não fumavam, $58,5 \%$ faziam uso de bebida alcoólica. Quanto à autopercepção do estado de saúde, 46,3\% percebiam sua saúde como sendo muito boa, $43,9 \%$ como boa e 9,8\% como excelente. Um indivíduo relatou possuir hipertensão arterial, um apontou doença cardíaca e um terceiro indicou ter doença pulmonar. Os policiais apresentaram valores pressóricos médios sistólicos e diastólicos de $117,8 \pm 8,8 \mathrm{mmHg}$ e $75,4 \pm 6,4 \mathrm{mmHg}$, respectivamente. Dois sujeitos apresentaram valores pressóricos sistólicos e diastólicos alterados ( $\geq 140 \mathrm{mmHg}$ e $\geq 90 \mathrm{mmHg}$, respectivamente). Quanto à prática de $\mathrm{AF}, 89,5 \%$ dos indivíduos foram considerados suficientemente ativos (média $=721,3 \pm$ $657,4 \mathrm{~min} / \mathrm{sem}$ ).

Em relação ao IMC, 23 (56,1\%) PRF estavam com pré-obesidade e $11(26,8 \%)$ apresentavam obesidade grau I. Em relação à circunferência da cintura $75,6 \%$ apresentavam risco aumentado para doenças cardiovasculares. Quanto ao \%GC, apenas nove (22\%) policiais ficaram na faixa considerada saudável. Nas variáveis neuromusculares, predominaram classificações ruim $(43,9 \%)$ para flexibilidade e fraco $(70,7 \%)$ na força de membros inferiores. Já a força isométrica de preensão manual apresentou valores classificatórios dentro da média para ambas as mãos. Estes e os demais resultados dos testes estão descritos na Tabela 1 .

Tabela 1. Medidas descritivas das variáveis de ordem nutricional, antropométrica e de aptidão física dos policiais rodoviários federais participantes do Programa Patrulha da Saúde, em Pelotas, Rio Grande do Sul, 2015.

\begin{tabular}{|c|c|c|c|}
\hline Variáveis & Média \pm DP (IC95\%) & $\mathbf{n}$ & $\%$ \\
\hline Índice de massa corporal & $28,5 \pm 3,4(27,5-29,6)$ & & \\
\hline Normal $(18,5-24,9)$ & & 6 & 14,3 \\
\hline Pré-Obesidade $(25,0-29,9)$ & & 23 & 56,1 \\
\hline Obesidade Grau I $(30,0-34,9)$ & & 11 & 26,8 \\
\hline Obesidade Grau II $(35,0-39,9)$ & & 1 & 2,4 \\
\hline Circunferência de cintura $(\mathrm{cm})$ & $99,2 \pm 8,5(96,6-101,9)$ & & \\
\hline Normal $(<94)$ & & 10 & 24,4 \\
\hline Risco aumentado ( $\geq 94)$ & & 31 & 75,6 \\
\hline Gordura corporal (\%) & $25,1 \pm 4,9(23,5-26,6)$ & & \\
\hline Saudável & & 9 & 22 \\
\hline Excesso de Gordura & & 16 & 39 \\
\hline Obeso & & 16 & 39 \\
\hline Flexibilidade $(\mathrm{cm})$ & $21,2 \pm 9,4(18,3-24,2)$ & & \\
\hline Ruim & & 18 & 43,9 \\
\hline Abaixo da média & & 5 & 12,2 \\
\hline Média & & 12 & 29,3 \\
\hline Acima da média & & 6 & 14,6 \\
\hline Força isométrica de preensão manual direita (kgf) & $47,4 \pm 6,2(45,5-49,4)$ & & \\
\hline Fraco & & 5 & 12,2 \\
\hline Abaixo da média & & 13 & 31,7 \\
\hline Médio & & 22 & 53,7 \\
\hline Bom & & 1 & 2,4 \\
\hline Força isométrica de preensão manual esquerda (kgf) & $44,6 \pm 6,3(42,6-46,6)$ & & \\
\hline Fraco & & 8 & 19,5 \\
\hline Abaixo da média & & 7 & 17 \\
\hline Médio & & 22 & 53,7 \\
\hline Bom & & 4 & 9,8 \\
\hline Força de membros inferiores (kgf) & $127,1 \pm 20,8(120,5-133,6)$ & & \\
\hline Fraco & & 29 & 70,7 \\
\hline Abaixo da média & & 9 & 22 \\
\hline Médio & & 3 & 7,3 \\
\hline Functional movement screening (pontos) $(n=26)^{*}$ & $13,8 \pm 2,6(12,7-14,9)$ & & \\
\hline$\leq 14$ pontos & & 15 & 57,7 \\
\hline$>14$ pontos & & 11 & 42,3 \\
\hline
\end{tabular}

DP, desvio padrão; IC, intervalo de confiança.

* Não foi possível avaliar todos os policiais da amostra em virtude da incompatibilidade entre o tempo necessário para esta avaliação e a disponibilidade dos sujeitos. 
Os participantes mais jovens apresentavam média menor de $\% \mathrm{GC}(23,3 \pm 5,4 \%)$ do que os mais velhos $(27,1 \pm 3,2 \%)(p<0,01)$ e também menor proporção de indivíduos com \%GC elevada $(\mathrm{p}<0,01)$. A análise da proporção entre as variáveis em relação à idade e o tempo de serviço, indica que os PRF mais velhos, quando estratificados pelo IMC, encontravam-se em maior proporção nas categorias de pré-obesidade e obesidade grau I, quando comparados aos mais novos $(p=0,04)$. Quanto à $C C$, policiais mais velhos $(p<0,01)$ e os mais antigos na instituição $(p=0,04)$ apresentaram predominância na classificação de risco superior para doenças cardiovasculares (Tabela 2).

A Tabela 3 apresenta a distribuição, por região anatômica, dos sintomas musculoesqueléticos responsáveis por afastamentos do trabalho no último ano.

Tabela 2. Comparação das variáveis de ordem nutricional, antropométricas e de aptidão física, divididos por idade e tempo de serviço dos policiais rodoviários federais participantes do Programa Patrulha da Saúde, em Pelotas, Rio Grande do Sul, 2015.

\begin{tabular}{|c|c|c|c|c|c|c|}
\hline \multirow[b]{2}{*}{ Variáveis } & \multicolumn{2}{|c|}{ Idade } & \multirow[b]{2}{*}{$\mathbf{P}$} & \multicolumn{2}{|c|}{ Tempo de serviço } & \multirow[b]{2}{*}{$\mathbf{p}$} \\
\hline & $\begin{array}{c}30 \text { a } 41 \text { anos } \\
n=22 *\end{array}$ & $\begin{array}{c}42 \text { a } 52 \text { anos } \\
n=19^{*}\end{array}$ & & $\begin{array}{c}2 \text { a } 10 \text { anos } \\
n=22^{*}\end{array}$ & $\begin{array}{c}11 \text { a } 21 \text { anos } \\
n=19 *\end{array}$ & \\
\hline IMC & & & $0,04^{+}$ & & & $0,86^{+}$ \\
\hline Normal & $24,1 \pm 0,7(27,3)$ & $0 \pm 0(0)$ & & $24,0 \pm 0,9(18,2)$ & $24,2 \pm 0,7(10,5)$ & \\
\hline Pré-Obesidade & $27,0 \pm 1,3(50,0)$ & $28,0 \pm 1,7(63,2)$ & & $27,1 \pm 1,5(54,6)$ & $28,0 \pm 1,8(57,9)$ & \\
\hline Obesidade Grau I & $32,9 \pm 0,7(18,2)$ & $31,6 \pm 0,8(36,8)$ & & $32,3 \pm 1,4(22,7)$ & $31,9 \pm 0,6(31,6)$ & \\
\hline Obesidade Grau II & $38,7 \pm 0(4,6)$ & $0 \pm 0(0)$ & & $38,7 \pm 0(4,6)$ & $0 \pm 0(0)$ & \\
\hline Circunferência da cintura (cm) & & & $<0,01 \mp$ & & & $0,04^{\ddagger}$ \\
\hline Normal & $90,1 \pm 4,0(50,0)$ & $93,0 \pm 1,4(10,5)$ & & $90,5 \pm 3,9(45,5)$ & $90,8 \pm 4,6(15,8)$ & \\
\hline Superior & $104,0 \pm 8,5(50,0)$ & $102,8 \pm 5,7(89,5)$ & & $104,5 \pm 7,8(54,5)$ & $102,4 \pm 6,1(84,2)$ & \\
\hline Gordura corporal (\%) & & & $0,05 \ddagger$ & & & $0,24^{\ddagger}$ \\
\hline Saudável & $17,7 \pm 2,0(36,4)$ & $19,3 \pm 0(5,3)$ & $0,05^{+}$ & $17,4 \pm 2,0(31,8)$ & $19,6 \pm 0,1(10,5)$ & $0,28^{+}$ \\
\hline Excesso de Gordura & $23,3 \pm 1,9(31,8)$ & $25,2 \pm 1,1(47,4)$ & & $23,7 \pm 2,0(31,8)$ & $24,9 \pm 1,5(47,4)$ & \\
\hline Obeso & $29,6 \pm 2,9(31,8)$ & $29,9 \pm 1,1(47,4)$ & & $29,5 \pm 2,7(36,4)$ & $30,0 \pm 1,1(42,1)$ & \\
\hline Flexibilidade (cm) & & & $0,6 \neq$ & & & $0,4^{\ddagger}$ \\
\hline Ruim & $13,2 \pm 7,0(50,0)$ & $11,1 \pm 3,2(36,8)$ & $0,6^{+}$ & $10,9 \pm 6,5(50,0)$ & $14,9 \pm 3,4(36,8)$ & $0,4^{+}$ \\
\hline Abaixo da média & $25,0 \pm 0,7(9,1)$ & $21,8 \pm 1,8(15,8)$ & & $24,5 \pm 0(4,6)$ & $22,8 \pm 2,3(21,1)$ & \\
\hline Média & $29,3 \pm 1,8(22,7)$ & $26,0 \pm 1,4(36,8)$ & & $28,9 \pm 1,9(27,3)$ & $25,8 \pm 1,5(31,6)$ & \\
\hline Acima da média & $36,1 \pm 0,7(18,2)$ & $29,8 \pm 0,4(10,5)$ & & $36,1 \pm 0,7(18,2)$ & $29,8 \pm 0,4(10,5)$ & \\
\hline FIPM direita (kgf) & & & $0,95^{+}$ & & & $0,95^{+}$ \\
\hline Fraco & $34,0 \pm 0(9,1)$ & $40,0 \pm 0(15,8)$ & & $34,0 \pm 0(9,1)$ & $40,0 \pm 0(15,8)$ & \\
\hline Abaixo da média & $44,6 \pm 1,5(31,8)$ & $42,5 \pm 3,1(31,6)$ & & $44,4 \pm 1,8(31,8)$ & $42,7 \pm 3,0(31,6)$ & \\
\hline Médio & $51,7 \pm 3,2(54,6)$ & $50,7 \pm 3,0(52,6)$ & & $51,3 \pm 3,3(54,6)$ & $51,1 \pm 3,0(52,6)$ & \\
\hline Bom & $62,0 \pm 0(4,6)$ & $0 \pm 0(0)$ & & $62,0 \pm 0(4,6)$ & $0 \pm 0(0)$ & \\
\hline FIPM esquerda (kgf) & & & $0,84^{+}$ & & & $0,26^{+}$ \\
\hline Fraco & $35,5 \pm 2,5(18,2)$ & $36,5 \pm 1,9(21,1)$ & & $34,7 \pm 2,3(13,6)$ & $36,8 \pm 1,8(26,3)$ & \\
\hline Abaixo da média & $40,0 \pm 0(13,6)$ & $41,0 \pm 1,2(21,1)$ & & $40,0 \pm 0(9,1)$ & $40,8 \pm 1,1(26,3)$ & \\
\hline Médio & $46,3 \pm 2,5(54,6)$ & $47,2 \pm 3,0(52,6)$ & & $46,6 \pm 2,3(63,6)$ & $46,9 \pm 3,5(42,1)$ & \\
\hline Bom & $56,7 \pm 1,2(13,6)$ & $58,0 \pm 0(5,3)$ & & $56,7 \pm 1,2(13,6)$ & $58,0 \pm 0(5,3)$ & \\
\hline Força de MMII (kgf) & & & $0,88^{+}$ & & & $1^{+}$ \\
\hline Fraco & $115,2 \pm 10,1(72,7)$ & $117,2 \pm 11,3(68,4)$ & & $115,2 \pm 9,6(68,2)$ & $117,1 \pm 11,7$ & \\
\hline Abaixo da média & $149,2 \pm 6,4(22,7)$ & $143,0 \pm 4,8(21,1)$ & & $149,2 \pm 6,4(22,7)$ & $143,0 \pm 4,8(21,1)$ & \\
\hline Médio & $165,0 \pm 0(4,6)$ & $180,0 \pm 0(10,5)$ & & $172,5 \pm 10,6(9,1)$ & $180,0 \pm 0(5,3)$ & \\
\hline FMS (pontos) & & & $0,6 \neq$ & & & $0,32^{\ddagger}$ \\
\hline$\leq 14$ pontos & $12,6 \pm 2,8(53,3)$ & $12,0 \pm 1,8(63,6)$ & & $12,5 \pm 2,7(50,0)$ & $12,1 \pm 2,0(70,0)$ & \\
\hline$>14$ pontos & $15,9 \pm 1,5(46,7)$ & $15,8 \pm 1,5(36,4)$ & & $15,8 \pm 1,4(50,0)$ & $16,0 \pm 1,7(30,0)$ & \\
\hline
\end{tabular}

* Os números iguais nas categorias de idade e de tempo de serviço não indicam que sejam os mesmos sujeitos em cada categoria de cada variável.

† Teste exato de Fisher; ₹ qui-quadrado.

IMC, índice de massa corporal; FIPM, força isométrica de preensão manual; MMII, membros inferiores; FMS, functional movement screening. 
Tabela 3. Distribuição, por região anatômica, dos sintomas musculoesqueléticos e dos afastamentos do trabalho no último ano entre policiais rodoviários federais participantes do Programa Patrulha da Saúde, em Pelotas, Rio Grande do Sul, 2015.

\begin{tabular}{|c|c|c|c|c|c|c|}
\hline \multirow{3}{*}{ Região anatômica } & \multicolumn{4}{|c|}{ Sintomas } & \multirow{2}{*}{\multicolumn{2}{|c|}{$\begin{array}{l}\text { Afastamentos do } \\
\text { trabalho no último ano }\end{array}$}} \\
\hline & \multicolumn{2}{|c|}{12 meses precedentes } & \multicolumn{2}{|c|}{7 dias precedentes } & & \\
\hline & n & $\%$ & n & $\%$ & $\mathbf{n}$ & $\%$ \\
\hline Ombros & 12 & 29,3 & 7 & 17,1 & 4 & 9,8 \\
\hline Pescoço & 17 & 41,5 & 5 & 12,2 & 1 & 2,4 \\
\hline Punhos, mãos, dedos & 11 & 26,8 & 7 & 17,1 & 2 & 4,9 \\
\hline Região dorsal & 9 & 22,0 & 5 & 12,2 & 2 & 4,9 \\
\hline Antebraço & 3 & 7,3 & 2 & 4,9 & 1 & 2,4 \\
\hline Região lombar & 17 & 41,5 & 11 & 26,8 & 6 & 14,6 \\
\hline Joelhos & 17 & 41,5 & 8 & 19,5 & 9 & 22,0 \\
\hline Tornozelos e pés & 8 & 19,5 & 2 & 4,9 & 4 & 9,8 \\
\hline Cotovelos & 4 & 9,8 & 2 & 4,9 & 3 & 7,3 \\
\hline Quadris e coxas & 5 & 12,2 & 3 & 7,3 & 3 & 7,3 \\
\hline
\end{tabular}

\section{DISCUSSÃO}

Esta investigação avaliou o perfil de PRF participantes do PPS em Pelotas, verificando a ocorrência de variáveis de ordem nutricional, antropométrica e neuromuscular em grupos com idades e tempos de serviço diferentes. Como principal achado, indica-se que, apesar de alto percentual de profissionais fisicamente ativos no lazer, e elevada percepção positiva de saúde, os participantes do estudo exibiam IMC e CC elevados, valores baixos de aptidão física, e alta prevalência de distúrbios musculoesqueléticos.

Quase a totalidade do efetivo do presente estudo informou não fumar atualmente, o que corrobora com resultados obtidos no estudo com policiais militares (PM) do Comando de Policiamento de Recife, Pernambuco [1]. Porém, os PRF de Pelotas apresentaram prevalência de fumantes acima da encontrada nos PM de Gurupi, Tocantins (5\%) [20]. Sabe-se que o tabagismo é fator de risco para o desenvolvimento de doenças cardiovasculares e câncer de pulmão, e sua cessação é responsável pela redução considerável do risco de morte [20]. Já a proporção de indivíduos que ingeriam bebida alcoólica foi superior à observada em um estudo realizado com policiais indianos (48\%) [5].

De forma semelhante ao estudo com PM de Santa Catarina [21], a maioria dos quais percebeu sua saúde como boa, mais da metade dos PRF da delegacia de Pelotas perceberam sua saúde como sendo muito boa ou excelente. Nesse contexto, estudo com participantes brasileiros adultos indicou que pessoas mais ativas tendem a classificar sua própria saúde de forma mais positiva do que aquelas que não são suficientemente ativas [22]. Os valores pressóricos médios de repouso, sistólico e diastólico dos participantes do presente estudo se encontravam dentro da normalidade, e apenas dois policiais apresentavam hipertensão arterial, sendo que apenas um PRF autorrelatou apresentar hipertensão. Já em um estudo com 296 policiais da Índia, a presença de hipertensão arterial associou-se a valores aumentados de IMC e CC [23].

Percentual restrito de policiais não atingiu as recomendações internacionais quanto à prática de $\mathrm{AF}$, com valores inferiores aos observado em PM de Recife-PE (73\%). Entretanto, o critério de classificação do NAF foi diferente do utilizado no presente estudo, pois não consideravam o tempo de caminhada para o cálculo de AF semanal [1]. Ressalta-se que elevados NAF e de aptidão são essenciais para manutenção da saúde, bem como para permitir que os indivíduos executem com eficácia suas tarefas laborais [11].

Outros estudos brasileiros encontraram altas prevalências de sobrepeso e obesidade, bem como CC com risco aumentado de doença cardiovascular entre policiais $[11,24]$. Perímetro de cintura aumentado é considerado melhor estimador da gordura visceral quando comparado com massa corporal, IMC ou relação cintura/quadril [24]. Os resultados do presente estudo não se mostram diferentes aos de uma revisão sistemática recente, que apresentou dados preocupantes sobre diferentes indicadores de 
obesidade entre policiais [25]. Dados referentes à relação da idade e do tempo de serviço com variáveis de ordem nutricional e antropométricas demonstraram que policiais mais velhos e com maior tempo de serviço se encaminham para perfis com maior risco para doenças cardiovasculares, como IMC acima de 25 e CC $\geq 94 \mathrm{~cm}$, o que corrobora com recente estudo com PM da Paraíba, em que o maior tempo de serviço esteve associado à elevação da CC [26]. Neste contexto, intervenções futuras poderiam voltar sua atenção a este grupo de policiais, para melhor manejo do processo saúde-doença no ambiente laboral.

A flexibilidade é uma característica que auxilia na execução de tarefas relacionadas ao trabalho [4]. Os índices de flexibilidade obtidos pelos PRF da presente amostra são preocupantes, visto que estão bem abaixo dos padrões recomendados para a população em geral, bem como quando comparados aos resultados de linha de base $(28,4 \mathrm{~cm})$ de um estudo com indivíduos ingressantes em uma academia de polícia nos Estados Unidos da América [2]. Tais dados indicam que a flexibilidade poderia passar a ser avaliada nos exames de capacidade física dessa força policial.

A força isométrica de preensão manual tem sido considerada preditora de mortalidade [27], e seu desempenho apontou valores médios, porém insuficientes quando comparados com estudo recente, o qual observou $53 \mathrm{~kg}$ e $50 \mathrm{~kg}$ para as mãos direita e esquerda, respectivamente [2]. Também tem sido demostrada associação entre a força de preensão manual e desempenho em tarefas táticas defensivas e de precisão do tiro [28], o que reforça a relevância do treinamento de força de membros superiores para as populações policiais. A força de membros inferiores também demonstrou níveis preocupantes, visto que grande parte ficou classificada com valores fracos, e sua média geral está inferior ao observado em estudo que investigou relações entre força, potência e velocidade de policiais da Special Weapons And Tactics (SWAT), uma unidade de polícia especializada [29].

O FMS tem sido considerado como ferramenta que oferece abordagem preventiva de lesões e preditora de desempenho do aparelho locomotor, identificando limitações/assimetrias [19], e a maior parte dos PRF estavam abaixo dos escores de referência para menor risco de lesão [3]. A pontuação total do FMS apresentou valores semelhantes aos de outro estudo com recrutas policias [3], sendo que um dos movimentos com pior desempenho, o rotary stability, pode ter relação com os principais locais de lesão nesta população, quais sejam, joelho e costas e, portanto, intervenções deveriam considerar estas regiões com maior atenção.
Quanto aos sintomas musculoesqueléticos apontados pelos PRF de Pelotas nos últimos 12 meses, destaca-se que sintomas semelhantes foram apontados em um estudo com 262 PM, no qual região lombar, costas, pescoço e joelhos foram apontados, a partir do mesmo instrumento, com prevalência de dor nos últimos 12 meses e percentuais semelhantes no registro dos sintomas nos últimos sete dias [6], os quais podem estar associados com a sobrecarga dos equipamentos utilizados pelos PRF durante o labor [4]. Assim, equipamentos mais leves e coletes mais flexíveis poderiam ser opções para reduzir os distúrbios músculoesqueléticos nesta população. Ademais, destaca-se que a parte inferior das costas e os joelhos estavam, também, dentre os principais sintomas geradores de afastamento do trabalho, o que pode causar impacto socioeconômico e outros prejuízos.

Algumas limitações do presente estudo devem ser citadas, tais como a coleta dos dados ter sido feita a partir de treinamento teórico e prático de apenas um dia e com profissionais que não tinham tanta experiência na área, apesar do acompanhamento constante dos pós-graduados em educação física; o uso do IPAQ como instrumento de avaliação no NAF, visto que pode apresentar valores superestimados de NAF a partir da auto aplicação de sua versão curta [30]; e a não consideração de fatores de confusão nas análises estatísticas. Por fim, sugere-se estudos futuros investigando delegacias da Polícia Rodoviária Federal em outras regiões, a fim de se obter informações globais acerca do perfil dos PRF, para que se possam identificar as condições de saúde e físicas desses profissionais e, com isso, orientar e reorganizar programas voltados à saúde e ao bemestar dos trabalhadores nas instituições de segurança pública.

Conclui-se que os PRF participantes do PPS em Pelotas, mesmo declarando estarem fisicamente ativos, apresentaram frequentemente condições morfológicas e físicas inadequadas, assim como níveis de força e flexibilidade inferiores aos recomendados para essa população. Nesta amostra, a idade mais avançada e maior tempo de serviço na Polícia Rodoviária Federal estiveram associados a parâmetros morfológicos de alto risco para doenças cardiovasculares. Observou-se, também, que os PRF possuíam alto risco de lesões musculoesqueléticas e afastavam-se trabalho principalmente por queixas osteomusculares nas regiões lombar e dos joelhos. Desta forma, sugere-se que os gestores utilizem a contribuição desses programas para suas corporações e, consequentemente, estimulem as práticas, na estrutura de trabalho, que possam reduzir 
os custos com tratamento de saúde, reduzindo assim o absenteísmo e aumentando a produtividade dos profissionais.

\section{NOTAS}

\section{Agradecimentos}

Ao Departamento de Polícia Rodoviária Federal; aos policiais da 7a Delegacia, em Pelotas; aos agentes apoiadores do programa Patrulha da Saúde.

\section{Apoio financeiro}

Academia Nacional da Polícia Rodoviária Federal (ANPRFP); Programa de Saúde do Servidor (PROSSERV); Patrulha da Saúde - programa de avaliação e monitoramento da saúde dos policiais rodoviários federais.

\section{Declaração de conflito de interesses}

Os autores declaram não haver conflitos de interesses relevantes ao conteúdo deste estudo, informam ter tido acesso a todos os dados obtidos e assumem completa responsabilidade pela integridade dos resultados.

\section{REFERÊNCIAS}

1. Ferreira DKS, Bonfim C, Augusto LGS. Fatores associados ao estilo de vida de policiais militares. Ciênc Saúde Colet. 2011;16(8):3403-12. https://doi.org/10.1590/S1413-81232011000900007

2. Crawley AA, Sherman RA, Crawley WR, Cosio-Lima L. Physical Fitness of Police Academy Cadets: Baseline Characteristics and Changes during a 16-Week Academy. J Strength Cond Res. 2016 May;30(5):1416-24. https://doi.org/10.1519/ JSC.0000000000001229

3. Bock C, Stierli M, Hinton B, Orr R. The Functional Movement Screen as a predictor of police recruit occupational task performance. J Bodyw Mov Ther. 2016 Apr;20(2):310-5. https://doi.org/10.1016/j.jbmt.2015.11.006

4. Pryor RR, Colburn D, Crill MT, Hostler DP, Suyama J. Fitness characteristics of a suburban special weapons and tactics team. J Strength Cond Res. 2012;26(3):752-7. https://doi.org/10.1519/JSC.0b013e318225f177

5. Thayyil J, Jayakrishnan TT, Raja M, Cherumanalil JM. Metabolic syndrome and other cardiovascular risk factors among police officers. N Am J Med Sci. 2012 Dec;4(12):630-5. https://doi.org/10.4103/1947-2714.104313

6. Da Trindade APNT, Oliveira LCN, Santos BMO, Oliveira FB, Quemelo PRV. Symptoms of musculoskeletal disorders among police officers. Arq Ciênc Saúde. 2015;22(2):42-5. https://doi.org/10.17696/2318-3691.22.2.2015.141

7. Orr RM, Stierli M. Injuries common to tactical personnel (A multidisciplinary review). Paper presented at: Tactical Strength and Conditioning Conference; 2013 Nov 8-10; Melbourne, Australia. Robina: Bond University; 2013.

8. Brasil. Ministério do Trabalho e Previdência Social - MTPS. Anuário Estatístico da Previdência Social - ano 2014 [Internet] Brasília; 2014 [cited 2016 Nov 27]. Avaliable from: http:/www.previdencia.gov.br/wp-content/uploads/2016/07/AEPS-2014.pdf

9. Elliot DL, Goldberg L, Kuehl KS, Moe EL, Breger RK, Pickering MA. The PHLAME (Promoting Healthy Lifestyles: Alternative Models' Effects) firefighter study: outcomes of two models of behavior change. J Occup Environ Med. 2007;49(2):204-13. https://doi.org/10.1097/JOM.0b013e3180329a8d

10. Kuehl KS, Elliot DL, MacKinnon DP, O’Rourke HP, DeFrancesco C, Miočević M, Valente M, Sleigh A, Garg B, McGinnis W, Kuehl H. The SHIELD (Safety \& Health Improvement: Enhancing Law Enforcement Departments) Study: Mixed Methods Longitudinal Findings. J Occup Environ Med. 2016 May;58(5):492-8. https://doi.org/10.1097/JOM.0000000000000716

11. Esteves JVDC, Andrade ML, Gealh L, Andreato LV, Franzói de Moraes SM. Caracterização da condição física e fatores de risco cardiovascular de policiais militares rodoviários. Rev Andal Med Deporte. 2014;7(2):66-71. https://doi.org/10.1016/S18887546(14)70064-4

12. Matsudo S, Araújo T, Matsudo V, Andrade D, Andrade E, Oliveira LC, Braggion G. International Physical Activity Questionnaire (IPAQ): study of validity and reliability in Brazil. Rev Bras Ativ Fis Saúde. 2001;6(2):5-18.

13. Pinheiro F, Bartholomeu T, Carvalho C. Validação do questionário nórdico de sintomas osteomusculares como medida de morbidade. Rev Saúde Pública. 2002;36(3):302-12. https://doi.org/10.1590/S0034-89102002000300008

14. World Health Organization. Obesity: preventing and managing the global epidemic. report of a WHO consultation (WHO technical report series 894). Geneva: WHO; 2000.

15. Kyle UG, Bosaeus I, De Lorenzo AD, Deurenberg P, Elia M, Gómez JM, Heitmann BL, Kent-Smith L, Melchior JC, Pirlich M, Scharfetter H, Schols AM, Pichard C; Composition of the ESPEN Working Group. Bioelectrical impedance analysis-part I: review of principles and methods. Clin Nutr. 2004 Oct;23(5):1226-43. https://doi.org/10.1016/j.clnu.2004.06.004

16. Gallagher D, Heymsfield SB, Heo M, Jebb SA, Murgatroyd PR, Sakamoto Y. Healthy percentage body fat ranges: an approach for developing guidelines based on body mass index. Am J Clin Nutr. 2000;72(3):694-701.

17. Canadian Standardized Test of Fitness (CSTF): Operations manual. 3rd ed. Ottawa: Government of Canada, Fitness and Amateur Sport; 1986.

18. American College of Sport Medicine (ACSM). Diretrizes do ACSM para os testes de esforço e sua prescrição. 3a ed. Rio de Janeiro: Guanabara Koogan; 2003.

19. Cook G, Burton L, Hoogenboom BJ, Voight M. Functional Movement Screening: the use of fundamental movements as an assessment of function—part 1. Int J Sports Phys Ther. 2014;9(3):396-409. 
20. Rezende AAB, Rodrigues ESR, Carlotto Herrera SDS, Silveira JM, Barreto KKS, Carmo PHF. Prevalência de tabagismo em policiais militares. Rev Méd Minas Gerais. 2012;22(2):146-52.

21. Silva R, Schlichting AM, Schlichting JP, Gutierres Filho PJ, Adami F, Silva A. Aspetos relacionados à qualidade de vida e atividade física de policiais militares de Santa Catarina - Brasil. Motricidade. 2012;8(3):81-9. https://doi.org/10.6063/motricidade.8(3).1159

22. Hallal PC, Victora CG, Wells JC, Lima RC. Physical inactivity: prevalence and associated variables in Brazilian adults. Med Sci Sports Exerc. 2003;35(11):1894-900. https://doi.org/10.1249/01.MSS.0000093615.33774.0E

23. Ganesh KS, Naresh AG, Bammigatti C. Prevalence and Risk Factors of Hypertension Among Male Police Personnel in Urban Puducherry, India. Kathmandu Univ Med J (KUMJ). 2014 Oct-Dec;12(48):242-6.

24. Tahan F, Pereira JC. Avaliação de risco cardiovascular por indicadores antropométricos em policiais militares de um batalhão do Sul de Minas Gerais. Nutr Bras. 2015;14(4):230-6.

25. Da Silva FC, Hernandez SS, Gonçalves E, Arancibia BA, Da Silva Castro TL, Da Silva R. Anthropometric indicators of obesity in policemen: a systematic review of observational studies. Int J Occup Med Environ Health. 2014 Dec;27(6):891-901. https://doi.org/10.2478/s13382-014-0318-0

26. Domingos-Gomes JR, Oliota-Ribeiro LS, Silva JS, Melo AC, Albuquerque Neto SL, Cirilo-Souza MS, Aniceto RR. Comparação da aptidão física relacionada à saúde e sua associação com o tempo de serviço entre policiais militares de operações especiais e de trânsito. J Phys Educ. 2016;27(1):1-12. https://doi.org/10.4025/jphyseduc.v27i1.2743

27. Rantanen T, Volpato S, Ferrucci L, Heikkinen E, Fried LP, Guralnik JM. Handgrip strength and cause-specific and total mortality in older disabled women: exploring the mechanism. J Am Geriatr Soc. 2003 May;51(5):636-41. https://doi.org/10.1034/j.16000579.2003.00207.x

28. Orr R, Stierli M, Hinton B, Steele M. Grip strength is associated with marksmanship and defensive tactics, but not injuries, in police recruits. Paper presented at: Australian Physiotherapy Association (APA) Conference; 2013 Oct 17-20; Melbourne, Australia. Robina: Bond University; 2013.

29. Dawes JJ, Orr R, Elder C, Krall K, Stierli M, Schilling B. Relationship between selected measures of power and strength and linear running speed amongst special weapons and tactics police officers. J Aust Strength Cond. 2015;23(3):23-8.

30. Hallal PC, Gomez LF, Parra DC, Lobelo F, Mosquera J, Florindo AA, Reis RS, Pratt M, Sarmiento OL. Lessons learned after 10 years of IPAQ use in Brazil and Colombia. J Phys Act Health. 2010 July;7 Suppl 2:S259-64. https://doi.org/10.1123/jpah.7. s2.s259 